\title{
IMPLANTAÇÃO DE UM PROGRAMA DE CONTROLE DE QUALIDADE DE UM ACELERADOR LINEAR DE 6 MeV DE FÓTONS*
}

\author{
Mafalda F. Berdaky', Linda V.E. Caldas²
}
Resumo Este trabalho apresenta a parte operacional do processo final envolvido na implantação de um programa de controle de qualidade por meio de testes rotineiros mecânicos e de radiação. 0 programa de controle de qualidade, durante 35 meses, mostrou a estabilidade excelente deste acelerador.
Unitermos: Acelerador linear. Controle de qualidade. Radiação de fótons.

Abstract Implementation of a quality control program for a $6 \mathrm{MeV}$ linear photon accelerator.

This paper describes the operational characteristics of the final process of implementation of a quality control program using routine mechanical and radiation tests. The quality control program was performed during 35 months and demonstrated the excellent stability of this accelerator.

Key words: Linear accelerator. Quality control. Photon radiation.

\section{INTRODUÇÃO}

À medida que a expectativa de vida da população aumenta, a incidência de câncer aumenta também na mesma proporção. Várias pesquisas de medicamentos e formas de tratamentos estão em andamento para se tentar a cura da doença. Uma delas, que data do início do século, é a radioterapia, que é um tratamento bastante eficaz no controle e até mesmo de cura da doença.

Logo após a descoberta dos raios $\mathrm{X}$ em 1895 por Roentgen, foi dado início a uma série de estudos e em 1898 Pierre e Marie Curie descobriram o rádio. Desde então, a radioterapia começou a se desenvolver. Em 29 de janeiro de 1896 foi tratado o primeiro paciente com radiação e em 1899 o primeiro caso de câncer; um epitelioma de células basais foi curado com radiação $0^{(\mathbf{1})}$.

O início da radioterapia no Brasil foi em 1901, no Rio Grande do Sul, com o médico Dr. Becker Pinto, que foi o primeiro a utilizar um aparelho de raios $\mathrm{X}$ para tratamento de um tumor de pele ${ }^{(2)} . \mathrm{O}$ primeiro acelerador linear do Brasil foi

* Trabalho realizado no Centro Brasileiro de Radioterapia, Oncologia e Mastologia (Cebrom), Goiânia, GO.

1. M.Sc., Departamento de Radioterapia do Cebrom.

2. Doutora, Instituto de Pesquisas Energéticas e Nucleares - Comissão Nacional de Energia Nuclear (Ipen-CNEN) São Paulo, SP.

Endereço para correspondência: Dra. Linda V.E. Caldas. Instituto de Pesquisas Energéticas e Nucleares, Comissão Nacional de Energia Nuclear. Caixa Postal 11.049. São Paulo, SP, 05422-970. E-mail: Icaldas@net.ipen.br

Aceito para publicação em 19/6/2001 instalado em 1972, no Hospital Alemão Oswaldo Cruz, em São Paulo(2).

A radioterapia é o tratamento por meio das radiações ionizantes, que utiliza doses de radiação altas e máquinas de energias altíssimas, em que qualquer erro no procedimento pode acarretar graves conseqüências ao paciente, inclusive a sua morte. Devido ao fato de se trabalhar com energias altas, necessita-se ter um controle da qualidade alto dessa radiação, para que a dose absorvida no volume alvo seja realmente a necessária. O erro máximo permitido na liberação dessa dose é de $5 \%{ }^{(3)}$.

$\mathrm{O}$ acelerador linear, antes de ser utilizado com pacientes, precisa passar por uma série de testes, chamados de testes de aceitação e de comissionamento. Os testes de aceitação devem ser executados em uma sequiência lógica, de maneira que os resultados de um teste não forcem uma mudança nos outros parâmetros do equipamento ${ }^{(4)}$. Nos testes de comissionamento são identificados todos os dados necessários à determinação de dose para pacientes e o equipamento é calibrado. Esta calibração consiste na determinação da relação entre a dose e a unidade monitora, à profundidade de dose máxima ${ }^{(5-7)}$.

Depois dos testes de comissionamento, o equipamento estará liberado para utilização no tratamento de pacientes, devendo-se tomar os devidos cuidados com os testes e os procedimentos de dosimetria de rotina, para a verificação da reprodutibilidade do sistema ${ }^{(5)}$. Existem vários protocolos e recomendações internacionais para uso em radioterapia ${ }^{(3,5-14)}$. Os protocolos mais utilizados no Brasil são o da American Association of Physicists in Medicine (AAPM) TG 21, de $1983^{(8)}$, e o da International Atomic Energy Agency (IAEA) No. 277, de 1987(6), revisado em $1996^{(7)}$.

Quando o equipamento está calibrado e os feixes da radiação caracterizados, o programa de controle de qualidade deve ser implementado, seguindo-se as recomendações internacionais ${ }^{(15,16)}$.

O objetivo deste trabalho é relatar os testes e apresentar os dados obtidos em um programa de controle de qualidade no Serviço de Radioterapia do Centro Brasileiro de Radioterapia, Oncologia e Mastologia (Cebrom), Goiânia, GO, e ainda acompanhar a reprodutibilidade de resposta do acelerador em questão.

\section{MATERIAIS E MÉTODOS}

\section{Sistema de radiação $X$}

$\mathrm{O}$ acelerador linear do Cebrom, com 6 $\mathrm{MeV}$ de energia, da Varian, modelo Clinac $600 \mathrm{C}$, permite a irradiação de pacientes com feixes de fótons, além de ser isocêntrico e possibilitar a irradiação em campos fixos e rotatórios; possui, ainda, os quatro colimadores assimétricos.

No sistema Clinac 600C a energia do feixe de fótons é $6 \mathrm{de} \mathrm{MeV}$, definida pela porcentagem de dose profunda (PDP) a $10 \mathrm{~cm}$ de profundidade na água, para um 
campo de $10 \times 10 \mathrm{~cm}^{2}$ e distância fontesuperfície de $100 \mathrm{~cm}$.

O equipamento possui duas câmaras contadoras, ou seja, um sistema duplo de dosimetria. O relógio de tratamento funciona com um sistema adicional de segurança para interrupção do feixe. O sistema de dosimetria controla a homogeneidade e a simetria do campo de tratamento.

\section{Sistema de medidas}

Foi utilizado sistema de medidas constituído por uma câmara de ionização à prova d'água, Exradin, Med Tec, modelo A12, EUA, e um eletrômetro Sun Nuclear Corporation, modelo 1010, EUA. Este sistema dosimétrico (câmara e eletrômetro) apresenta certificado de calibração de 21/12/99 do Instituto de Pesquisas Energéticas e Nucleares - Comissão Nacional de Energia Nuclear (Ipen-CNEN/SP).

Para as medidas de densidade óptica dos filmes radiográficos foi utilizado o densitômetro digital MRA, série CQ 010103, Brasil, e os filmes utilizados foram do Diagnostic Film Ready-Pack XOmat V da Kodak.

Foram utilizados os seguintes fantomas: a) fantoma de água, com dimensões de $30 \times 40 \times 40 \mathrm{~cm}^{3}$, com manivela manual para se realizar medidas na água em profundidade, Med Tec, modelo MT100, EUA; b) fantoma de água, com dimensões de $52 \times 65 \times 48 \mathrm{~cm}^{3}$, e sistema automático CRS ("computerized radiation scanner"), Dosimetrika, Brasil.

\section{RESULTADOS}

O programa de controle de qualidade de um acelerador deve levar em conta a necessidade dos testes a serem feitos, a freqüência com que eles devem ser realizados, as técnicas a serem seguidas, sempre lembrando que os equipamentos a serem utilizados para os testes devem ser de fácil manuseio e utilização para economizar tempo de trabalho.

Assim, no Cebrom foram implementados os seguintes testes, com as respectivas frequiências (entre parênteses):

1. Determinação do fator de calibração (semanal);

2. Verificação da energia do feixe (semanal);
3. Coincidência de campo luminoso $\times$ campo radioativo (mensal);

4. Diferença entre retículo e escala óptica (mensal);

5. Botões de segurança - botões de emergência (semanal);

6. Dimensões do campo luminoso indicado $\times$ medido (semanal);

7. Indicador de distância foco-superfície - escala óptica $\times$ indicador (semanal);

8. Indicador de ângulo da coluna (semanal);

9. Indicador de ângulo do colimador (semanal).

\section{Determinação do fator de calibração}

O fator de calibração é determinado tomando-se medidas na água a $5 \mathrm{~cm}$ de profundidade com a câmara de ionização, utilizando-se o protocolo americanoAAPMTG $21^{(8)}$. Na Tabela 1 podem ser observados os resultados obtidos.

Tabela 1 Fatores de calibração utilizando a taxa de dose de $320 \mathrm{cGy} / \mathrm{UM}$, onde UM é a unidade monitora.

\begin{tabular}{|l|ccc|}
\hline \multirow{2}{*}{ Mês } & \multicolumn{3}{|c|}{$\begin{array}{c}\text { Fator de calibração } \\
\text { (cGy/UM) }\end{array}$} \\
\cline { 2 - 4 } & 1998 & 1999 & 2000 \\
\hline Janeiro & - & 0,998 & 1,001 \\
Fevereiro & 1,000 & 0,996 & 1,000 \\
Março & 1,000 & 0,999 & 0,999 \\
Abril & 0,998 & 0,996 & 0,998 \\
Maio & 0,998 & 0,996 & 0,994 \\
Junho & 0,998 & 1,000 & 0,995 \\
Julho & 0,999 & 0,997 & 1,002 \\
Agosto & 0,998 & 0,994 & 0,995 \\
Setembro & 0,999 & 0,997 & 0,995 \\
Outubro & 1,000 & 0,998 & 0,990 \\
Novembro & 0,997 & 0,994 & 0,994 \\
Dezembro & 0,997 & 0,997 & 1,000 \\
\hline
\end{tabular}

Pode-se verificar que a variação máxima foi de 1,0\% em apenas um caso em relação ao valor unitário, sendo que a maioria se manteve dentro do valor esperado, mostrando que os resultados são satisfatórios.

\section{Verificação da energia do feixe}

A câmara de ionização é posicionada no centro de um campo de $10 \times 10 \mathrm{~cm}^{2}$ no fantoma de água, primeiramente à profundidade de $10 \mathrm{~cm}$ e depois à profundidade de $20 \mathrm{~cm}$, obtendo-se medidas com valores médios de $\mathrm{L}_{10}$ e $\mathrm{L}_{20}$, respectivamente. $\mathrm{O}$ desvio padrão máximo das medidas foi de $0,5 \%$.

A energia é verificada por: $\mathrm{L}_{20} / \mathrm{L}_{10}$.

A relação para este aparelho é de (67 $\pm 2) \%$. Na Tabela 2 são apresentados os valores obtidos.

Tabela 2 Resultados obtidos para o teste da verificação da energia do feixe de radiação.

\begin{tabular}{|l|ccc|}
\hline \multirow{2}{*}{ Mês } & \multicolumn{3}{|c|}{$\left(L_{20} L_{10}\right) 100(\%)$} \\
\cline { 2 - 4 } & 1998 & 1999 & 2000 \\
\hline Janeiro & - & 67,8 & 67,9 \\
Fevereiro & 66,9 & 67,7 & 67,9 \\
Março & 66,9 & 67,9 & 67,6 \\
Abril & 66,9 & 68,0 & 67,7 \\
Maio & 67,1 & 67,9 & 67,6 \\
Junho & 67,4 & 67,9 & 67,4 \\
Julho & 67,5 & 67,8 & 67,4 \\
Agosto & 67,6 & 68,1 & 67,5 \\
Setembro & 67,8 & 68,0 & 67,5 \\
Outubro & 67,8 & 67,6 & 67,5 \\
Novembro & 67,7 & 67,8 & 67,5 \\
Dezembro & 67,7 & 67,7 & 67,5 \\
\hline
\end{tabular}

Os resultados para este acelerador mostraram uma relação de energia com valor de $67,6 \%$ quando comparados com a literatura ${ }^{(17)}$, que é de $67,5 \%$; os resultados são, portanto, equivalentes.

\section{Coincidência de campo luminoso $\times$ campo radioativo}

Um filme dentro do envelope, no qual se marca um campo de $10 \times 10 \mathrm{~cm}^{2}$, é colocado entre duas placas de acrílico, sendo que a de baixo é para o retroespalhamento e a de cima, de $1,5 \mathrm{~cm}$, é para se obter a condição de equilíbrio eletrônico do aparelho. Este aparato é colocado à distância de $100 \mathrm{~cm}$ do foco e o filme é irradiado com uma dose de 50 cGy. Depois de irradiado, o filme é avaliado no densitômetro, no centro do campo e nas direções x+, x-, y+e y-. Quando a leitura em relação ao centro cair até $50 \%$, o campo é definido, e então pode-se comparar o tamanho do campo radioativo com o luminoso. A Tabela 3 apresenta os valores de variação do campo. 
Tabela 3 Resultados obtidos para o teste de coincidência de campo luminoso $\times$ campo radioativo.

\begin{tabular}{|l|ccr|}
\hline \multirow{2}{*}{ Mês } & \multicolumn{3}{|c|}{$\begin{array}{c}\text { Variação do campo } \\
\text { (mm) }\end{array}$} \\
\cline { 2 - 4 } & 1998 & 1999 & 2000 \\
\hline Janeiro & - & 2,0 & 1,0 \\
Fevereiro & - & 1,0 & 1,0 \\
Março & - & 1,0 & 0,5 \\
Abril & - & 1,0 & 1,0 \\
Maio & 2,0 & 1,0 & 1,0 \\
Junho & 1,0 & 0,5 & 1,0 \\
Julho & 1,0 & 0,5 & 1,0 \\
Agosto & 1,0 & 0,5 & 1,0 \\
Setembro & 1,0 & 1,0 & 2,0 \\
Outubro & - & 1,0 & 0,5 \\
Novembro & 0,5 & 1,0 & 1,0 \\
Dezembro & 1,0 & 1,0 & 0,5 \\
\hline
\end{tabular}

diação é exatamente o que se está utilizando durante as irradiações. As dimensões do campo foram examinadas no período de fevereiro de 1998 a dezembro de 2000.

Os resultados obtidos, conforme pode-se verificar na Tabela 4, sempre estiveram abaixo do limite recomendado pelo fabricante.

Os testes para os colimadores assimétricos têm-se mantido dentro do mesmo padrão.

\section{Indicador de distância foco-superfície}

Este teste é realizado com o objetivo de verificar a distância de tratamento utilizada. As distâncias são medidas com a escala óptica do aparelho e em seguida são examinadas com os respectivos indicadores mecânicos. Foram feitas verificações semanais durante o período de fevereiro de 1998 a dezembro de 2000, não tendo sido observada nenhuma variação entre os valores indicados e os medidos.

\section{Indicador de ângulo da coluna}

O objetivo deste teste é verificar as angulações mostradas no marcador digital do aparelho.

Os resultados estão apresentados na Tabela 5. A maior variação observada foi de $1,0^{\circ}$ em apenas um caso; todos os outros dados obtidos estão dentro das especificações. Os resultados são, portanto, satisfatórios.
Pode-se verificar que apenas em algumas vezes chegou-se a $2 \mathrm{~mm}$, que é o limite recomendado, mostrando que os resultados são satisfatórios.

\section{Diferença entre retículo e escala óptica}

Este teste constitui-se simplesmente da verificação de quanto o centro do campo do aparelho está deslocado com relação à escala óptica (que indica a distância foco-superfície). O teste foi realizado mensalmente, entre fevereiro de 1998 e dezembro de 2000 , não tendo sido detectada nenhuma variação.

\section{Botões de segurança - botões de emergência}

Os botões de segurança são acionados para se verificar o corte imediato da emissão de radiação. Com o aparelho na condição de estacionário ("stand-by"), aciona-se o botão de emergência e o aparelho deve desligar por completo. Há ainda o teste da porta, que é feito com o aparelho em funcionamento. Abre-se a porta e o feixe de radiação deve parar imediatamente. Nos dois casos, nunca houve nenhum tipo de falha mecânica no período entre 1998 e 2000.

\section{Dimensões do campo luminoso}

Este teste é realizado com a finalidade de verificar se o campo luminoso de ra-
Tabela 4 Resultados obtidos para dimensões do campo luminoso - indicado $\times$ medido

\begin{tabular}{|c|c|c|c|c|c|c|}
\hline \multirow{3}{*}{ Mês } & \multicolumn{6}{|c|}{ Campo luminoso $\left(\mathrm{cm}^{2}\right)$} \\
\hline & $10 \times 10$ & $20 \times 20$ & $10 \times 10$ & $20 \times 20$ & $10 \times 10$ & $20 \times 20$ \\
\hline & \multicolumn{2}{|c|}{1998} & \multicolumn{2}{|c|}{1999} & \multicolumn{2}{|c|}{2000} \\
\hline Janeiro & - & - & $10,0 \times 10,0$ & $19,9 \times 20,0$ & $10,0 \times 10,0$ & $20,1 \times 20,0$ \\
\hline Fevereiro & $10,0 \times 10,0$ & $20,0 \times 20,1$ & $10,0 \times 10,1$ & $20,1 \times 20,1$ & $10,0 \times 10,1$ & $20,0 \times 20,0$ \\
\hline Março & $10,0 \times 10,0$ & $20,0 \times 20,0$ & $10,0 \times 10,1$ & $20,1 \times 20,0$ & $10,0 \times 10,1$ & $20,0 \times 20,0$ \\
\hline Abril & $9,9 \times 10,0$ & $19,9 \times 20,0$ & $10,1 \times 10,0$ & $20,1 \times 20,0$ & $10,0 \times 10,1$ & $20,0 \times 20,1$ \\
\hline Maio & $10,2 \times 10,0$ & $20,2 \times 20,0$ & $10,0 \times 10,0$ & $20,1 \times 20,0$ & $10,0 \times 10,1$ & $20,0 \times 20,1$ \\
\hline Junho & $10,1 \times 10,0$ & $20,0 \times 19,9$ & $10,0 \times 10,0$ & $20,1 \times 20,0$ & $10,0 \times 10,1$ & $20,0 \times 20,1$ \\
\hline Julho & $10,1 \times 10,0$ & $19,9 \times 19,9$ & $10,0 \times 10,1$ & $20,1 \times 20,0$ & $10,0 \times 10,1$ & $20,0 \times 20,0$ \\
\hline Agosto & $10,0 \times 10,1$ & $20,0 \times 20,1$ & $10,1 \times 10,0$ & $20,1 \times 20,0$ & $10,0 \times 10,1$ & $20,0 \times 20,0$ \\
\hline Setembro & $10,1 \times 10,1$ & $20,0 \times 20,0$ & $10,0 \times 10,0$ & $20,1 \times 20,0$ & $10,0 \times 10,1$ & $20,0 \times 20,1$ \\
\hline Outubro & $10,0 \times 10,1$ & $20,0 \times 20,1$ & $10,0 \times 10,0$ & $20,1 \times 20,0$ & $10,1 \times 10,0$ & $20,1 \times 20,0$ \\
\hline Novembro & $10,0 \times 10,1$ & $20,1 \times 20,0$ & $10,1 \times 10,1$ & $20,1 \times 20,0$ & $10,1 \times 10,0$ & $20,0 \times 20,1$ \\
\hline Dezembro & $10,0 \times 10,1$ & $20,1 \times 20,0$ & $10,0 \times 10,0$ & $20,1 \times 20,0$ & $10,0 \times 9,9$ & $20,0 \times 20,0$ \\
\hline
\end{tabular}

Tabela 5 Resultados obtidos para os ângulos da coluna.

\begin{tabular}{|c|c|c|c|c|c|c|c|c|c|c|c|c|}
\hline \multirow{3}{*}{ Mês } & \multicolumn{12}{|c|}{ Posicionamento da coluna } \\
\hline & $0^{\circ}$ & $90^{\circ}$ & $180^{\circ}$ & $270^{\circ}$ & $0^{\circ}$ & $90^{\circ}$ & $180^{\circ}$ & $270^{\circ}$ & $0^{\circ}$ & $90^{\circ}$ & $180^{\circ}$ & $270^{\circ}$ \\
\hline & \multicolumn{4}{|c|}{1998} & \multicolumn{4}{|c|}{1999} & \multicolumn{4}{|c|}{2000} \\
\hline Janeiro & - & - & - & - & 0 & 89,7 & 179,7 & 270,1 & 0 & 90,0 & 179,8 & 270,1 \\
\hline Fevereiro & 0 & 90,5 & 180,0 & 270,0 & 0 & 90,0 & 180,1 & 270,0 & 0 & 90,1 & 180,0 & 270,1 \\
\hline Março & 0 & 90,5 & 180,0 & 270,0 & 0 & 90,1 & 180,0 & 270,1 & 0 & 90,1 & 180,0 & 270,1 \\
\hline Abril & 0 & 90,5 & 180,0 & 270,0 & 0 & 90,2 & 180,0 & 270,0 & 0 & 90,1 & 180,0 & 270,0 \\
\hline Maio & 0 & 90,5 & 180,0 & 270,0 & 0 & 90,0 & 180,0 & 270,1 & 0 & 90,0 & 180,0 & 270,1 \\
\hline Junho & 0 & 90,0 & 180,0 & 270,0 & 0 & 90,1 & 179,9 & 270,1 & 0 & 90,0 & 179,9 & 270,1 \\
\hline Julho & 0 & 90,0 & 180,0 & 270,0 & 0 & 90,0 & 180,1 & 269,9 & 0 & 90,1 & 179,8 & 270,1 \\
\hline Agosto & 0 & 90,0 & 180,0 & 270,0 & 0 & 90,2 & 180,2 & 270,4 & 0 & 90,0 & 179,9 & 270,0 \\
\hline Setembro & 0 & 90,0 & 181,0 & 270,0 & 0,1 & 90,1 & 180,0 & 269,9 & 0 & 90,1 & 179,9 & 270,0 \\
\hline Outubro & 0 & 90,0 & 180,5 & 270,0 & 0 & 90,1 & 180,1 & 270,0 & 0 & 90,1 & 179,8 & 270,0 \\
\hline Novembro & 0 & 90,0 & 180,0 & 270,1 & 0 & 90,1 & 180,1 & 270,1 & 0 & 90,2 & 179,7 & 270,1 \\
\hline Dezembro & 0 & 90,0 & 180,1 & 270,0 & 0 & 90,1 & 180,1 & 270,0 & 0 & 90,4 & 179,7 & 270,0 \\
\hline
\end{tabular}




\section{Indicador de ângulo do colimador}

O objetivo deste teste é verificar as angulações mostradas no marcador digital do aparelho.

Os resultados obtidos estão apresentados na Tabela 6. A maior variação foi de $1,0^{\circ} \mathrm{em}$ apenas dois meses e para um lado apenas, estando todos os outros dados obtidos dentro das especificações. Os resultados são, portanto, satisfatórios.

\section{CONCLUSÕES}

O sistema do acelerador do Cebrom, em Goiânia, entrou em funcionamento em fevereiro de 1998, e desde então foi implantado o programa de controle de qualidade descrito neste trabalho. Após dois anos e 11 meses de funcionamento, a maior variação verificada no fator de calibração foi de 1,0\% em apenas um caso, sendo que a maioria se manteve dentro das especificações. Quanto à energia, a maior variação foi de $0,9 \%$, sendo que na maioria dos casos ela esteve dentro das especificações, mostrando, assim, a excelente estabilidade do aparelho. A longo prazo, o programa de controle de qualidade em andamento deverá continuar, para que se possa manter a qualidade dos tratamentos de pacientes.

A cada três meses o aparelho passa por uma revisão completa de peças e acessórios, e quando se constata qualquer defeito o acessório e/ou a peça são substituídos.

Sempre que os botões de segurança e a porta foram acionados com o aparelho em funcionamento, o feixe foi interrompido imediatamente, mostrando que o aparelho apresenta a segurança desejada.

Os campos radioativo e luminoso têm ficado dentro de no máximo $1 \mathrm{~mm}$ de diferença, exceto em três casos em que ficaram em $2 \mathrm{~mm}$, mas que foram corrigidos imediatamente, conforme observado nos resultados apresentados.

Tabela 6 Resultados obtidos para os ângulos do colimador.

\begin{tabular}{|c|c|c|c|c|c|c|c|c|c|}
\hline \multirow{3}{*}{ Mês } & \multicolumn{9}{|c|}{ Posicionamento do colimador } \\
\hline & $0^{\circ}$ & $90^{\circ}$ & $270^{\circ}$ & $0^{\circ}$ & $90^{\circ}$ & $270^{\circ}$ & $0^{\circ}$ & $90^{\circ}$ & $270^{\circ}$ \\
\hline & \multicolumn{3}{|c|}{1998} & \multicolumn{3}{|c|}{1999} & \multicolumn{3}{|c|}{2000} \\
\hline Janeiro & - & - & - & 0,1 & 90,1 & 270,5 & 0 & 90,0 & 270,0 \\
\hline Fevereiro & 0 & 90,5 & 270,5 & 0 & 90,0 & 270,0 & 0 & 90,2 & 270,2 \\
\hline Março & 0,5 & 89,5 & 269,1 & 0 & 90,2 & 270,2 & 0 & 90,1 & 270,1 \\
\hline Abril & 0 & 90,5 & 271,0 & 0 & 90,2 & 270,2 & 0 & 90,2 & 270,2 \\
\hline Maio & 0 & 90,9 & 271,0 & 0 & 90,0 & 270,2 & 0 & 90,0 & 270,2 \\
\hline Junho & 0 & 90,0 & 270,0 & 0 & 90,1 & 270,2 & 0 & 90,1 & 270,2 \\
\hline Julho & 0 & 90,0 & 270,0 & 0,1 & 90,0 & 269,2 & 0 & 90,1 & 270,0 \\
\hline Agosto & 0 & 90,0 & 270,0 & 0 & 90,2 & 270,2 & 0 & 90,1 & 270,2 \\
\hline Setembro & 0 & 90,0 & 270,1 & 0,1 & 90,1 & 270,4 & 0 & 90,1 & 270,3 \\
\hline Outubro & 0 & 90,0 & 270,0 & 0 & 90,2 & 270,1 & 0 & 90,1 & 270,4 \\
\hline Novembro & 0 & 90,1 & 270,1 & 0 & 90,0 & 270,2 & 0 & 90,2 & 270,5 \\
\hline Dezembro & 0 & 90,1 & 270,1 & 0 & 90,1 & 270,0 & 0 & 90,1 & 270,1 \\
\hline
\end{tabular}

\section{Agradecimento}

As autoras agradecem ao Conselho Nacional de Desenvolvimento Científico e Tecnológico (CNPq), pelo apoio financeiro parcial.

\section{REFERÊNCIAS}

1. Bentel GC. Radiation therapy planning. 2nd ed. New York: McGraw-Hill, 1996.

2. Pinto ACLC. A radioterapia no Brasil. $2^{\mathrm{a}}$ ed. Curitiba: Sociedade Brasileira de Radioterapia, 2000.

3. International Commission on Radiation Units and Measurements. Measurement of absorbed dose in a phantom irradiated by a single beam of $\mathrm{X}$ or gamma rays. ICRU Report No. 23, Bethesda, MD, 1973.

4. Scaff LAM. Física da radioterapia. São Paulo: Sarvier, 1997.

5. Nath R, Biggs PJ, Bova FJ, et al. AAPM code of practice for radiotherapy accelerators. Report of AAPM Radiation Therapy Task Group No. 45. Med Phys 1994;21:1093-121.

6. International Atomic Energy Agency. Absorbed dose determination in photon and electron beams: an international code of practice. IAEA, Technical Reports Series No. 277, Vienna, 1987.

7. International Atomic Energy Agency. Review of data and methods recommended in the international code of practice. IAEA Technical Reports Series No. 277, Absorbed dose determination in photon and electrom beams. IAEA, Tecdoc-897, Vienna, 1996. 8. American Association of Physicists in Medicine.
A protocol for the determination of absorbed dose from high-energy photon and electron beams. Task Group No. 21. Med Phys 1983;10:741-71.

9. Andreo P, Burns DT, Hohlfeld K, et al. An international code of practice for radiotherapy dosimetry based on absorbed dose to water standards. Radiother Oncol 1999;51(suppl.1).

10. International Atomic Energy Agency. Design and implementation of a radiotherapy programme: clinical, medical physics, radiation protection and safety aspects. IAEA, Tecdoc-1040, Vienna, 1998.

11. International Atomic Energy Agency. Absorbed dose determination in external beam radiotherapy: an international code of practice for dosimetry based on standards of absorbed dose to water. IAEA, Technical Reports Series s/n, Vienna, 2000.

12. Perez CA. Principles and practice of radiation oncology. 3rd ed. Philadelphia, New York: Lippincott-Raven, 1998.

13. Williams JR, Thwaites DI. Radiotherapy physics in practice. 2nd ed. Oxford: Oxford Univ Press, 2000.

14. Andreo P. Dose determination with plane-parallel ionization chambers in therapeutic electron and photon beams. SSDL-Newsletter 1999;40:16.

15. International Atomic Energy Agency. Quality assurance in radiotherapy. IAEA, Tecdoc-989, Vienna, 1997.

16. International Atomic Energy Agency. Aspectos físicos de la garantía de calidad en radioterapia: protocolo de control de calidad. IAEA, Tecdoc-1151, Vienna, 2000.

17. Aird EGA, Burns JE, Day MJ, et al. Central axis depth dose data for use in radiotherapy. Br J Radiol 1996; (suppl. 25). 